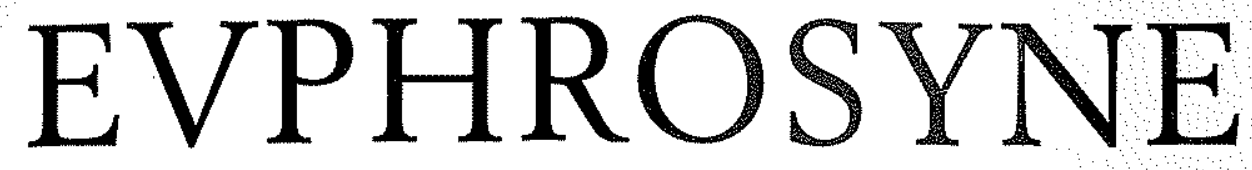

REVISTA DE FILOLOGIA CLÁSSICA

NOVA SERIE - VOLUME XL

SEPARATA

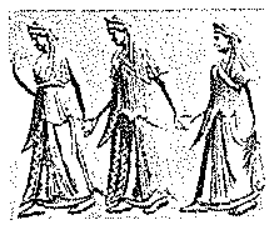




\title{
Dos pasajes de la conquista de Mallorca en la Chronica gestorum Iacobi I de Pere Marsili*
}

\author{
Marla Carmen Puche López \\ Universidad de Alicante \\ carmen.puche@ua.es
}

\section{Introducción}

Como es bien sabido, a lo largo del siglo XIII se produce una transformación sustancial de la práctica historiográfica catalana y, a partir de las últimas décadas del siglo, surge dentro de su tradición historiográfica una nueva modalidad de obras que, frente a otras anteriores redactadas en latín y traducidas posteriormente a lengua romance, están escritas ya originariamente en catalán y se caracterizan por un gran valor literario ${ }^{1}$. A este grupo de obras pertenece, junto con las crónicas de Bernat Desclot, Ramon Muntaner y Pedro el Ceremonioso (estas dos últimas ya de siglo XIV), el Llibre dels fets de Jaime I, en el que, de forma autobiográfica y a modo de libro de memorias, se relata la vida de Jaime I, desde su concepción, presentada como designio de la Providencia, hasta el momento de su muerte. Esta obra constituye sin duda un unicum dentro del grupo, tanto por los apasionados debates filológicos que ha suscitado (el grado de intervención directa del rey en la redacción de la obra, su posible origen épico y versificado, la filiación y jerarquización de los manuscritos etc. ${ }^{2}$ ) como $-y$, sobre todo-, por su origi-

* Este trabajo se inscribe en la línea de investigación desarrollada en el Grupo de Investigación CODOLVA de la Universidad de Alicante, dirigido por Dr. D. Juan F. Mesa Sanz.

1 Cf. J. Aurell, "From genealogies to chronicles: the power of the form in Medieval Catalan Historiography", Viator, 36, 2005, pp. 235-264, concretamente en pp. 251-252 y R. Ar.eMANY, "Literatura comparada: literatura castellana i catalana al segle XII", en G. CoLÓN DOMENECH, T. Martínez Romero (eds.), El rei Jaume $I$ : fets, actes $i$ paraules, Castello/Barcelona, 2008, pp. 167.181, concretamente en p. 176.

2 Si ya afirma F. Sol.pevila (Cronistes, joglars $i$ poetes, a cura de J. Molas i J. Massot i Muntaner, Barcelona, 1996, p. 75) que ninguna otra crónica presenta los problemas y dificultades que ofrece la de Jaime 1, J. M. PuJol da comienzo a su clarificador repaso de las diversas teorias en torno a la obra ("¿Cultura eclesiàstica o competència retorica? El llatí, la Bíblia i el rei En Jaume", Estudis romànics, XXIII, 2001, pp. 147-172, concretamente en p. 147) de esta manera: "Ha estat el P. Burns que ha dit que el Llibre del rei En Jaume és un document tan insolit per a l'època medieval que els dubtes sobre la seva autenticitat es presenten de manera 
nalidad y por la autenticidad y frescura que se percibe en la descripción de los intensos y gloriosos acontecimientos de un reinado que es narrado desde la perspectiva de su protagonista.

Un aspecto de referencia obligada en los estudios sobre la obra es la relación entre la versión catalana que conservamos del Llibre dels fets y la versión latina que de la misma realizó en 1313 el dominico Pere Marsili ${ }^{3}$ por encargo de Jaime II de Aragón, hijo de Pedro el Grande y nieto, por tanto, de Jaime $\mathrm{I}$.

En su aproximación a esta obra latina, la atención de los estudiosos se ha centrado fundamentalmente en establecer el orden de prelación entre ésta y el Llibre dels fets y, aunque la teoría de la influencia del texto latino sobre el texto catalán formulada por $\mathrm{M}$. de Montoliu${ }^{4}$, que tiene precedentes en los estudios de J. de Villarroya ${ }^{5}$ y $\mathrm{J}$. Villanueva ${ }^{6}$, ha tenido continuidad en críticos posteriores como Nicolau d'Olwer ${ }^{7}$, trabajos como los de MartinChabot $^{8}$, F. Soldevila ${ }^{9}$ o S. Asperti ${ }^{10}$ han defendido con sólidos argumentos la prelación del texto catalán sobre el latino, hasta el punto de que J. M. Pujol afirma que "El tema s'hauria de considerar ja tancat"11. Así pues, sin entrar en posibles diferencias entre la crónica de Jaime I previa a la versión de

natural." En la monografía de G. COLON DOMENECH, T. MARTINEz Romero (eds.): El rei Jaume I: fets, actes $i$ paraules, Castello/Barcelona, Fundació Germà Colon Domenech - Publicacions de l'Abadia de Montserrat, 2008, pueden consultarse sobre la crónica catalana trabajos como el de S. M. Cingolani, "El Llibre dels fets del rei Jaume i el Llibre del rei En Pere de Bernat Desclot", pp. 287-312; J. M. Pujol, "El programa narratiu del Llibre del rei En Jaume", pp. 257-286 o LL. MARTIN PAscuaz, "Angel Aguiló i la tradició manuscrita del Libre dels fets", pp. 335-354.

3 Es bien poco lo que sabemos de la vida de Marsill. La primera noticia que tenemos de el es de 1303, en la que se le menciona como miembro de la orden de Predicadores en el convento de Mallorca y, tras un silencio documental de 6 años, conocemos de su actividad como legado del monarca Jaime II ante el Papa Clemente $V$ a finales de 1309. El último dato que tenemos es del año 1316. Cf. Pere Marsier, Chronica gestorum invictissimi Domini lacobi primi Aragoniae regis, edición de M. ${ }^{2}$ D. Martínez San Pedro, Almería, 1984, pp. 17 y ss. y R. VINAS, A. Vinas, La conquête de Majorque. Textes et documents. Perpiñán, Société Agricole, Scientifique et Littéraire des Pyrénées Orientales, 2004, pp. 207 y s.

${ }^{4}$ M. DE.Monroliu, "La cançó de gesta de Jaume I: nova teoria sobre la cronica del Conqueridor", Butlletí Arqueològic: Publicació de la Reial Societat Arqueologica Tarraconense, ép. III, n. ${ }^{\circ}$ 8, 1922, pp. 177-186; ép. III, n. .9 , 1922, pp. 209-216; "La versión catalana y la latina de la Cronica de Jaime I, confrontadas", Butlleti Arqueologic: Publicació de la Reial Societat Arqueologica Tarraconense, ép. IV, 1952, pp. 89-103.

${ }^{5} \mathrm{~J}$. VillaArroya, Colección de cartas historico-críticas en que se convence que el rey D. Jayme I de Aragon no fue el verdadero autor de la Cronica o Comentarios que corren en su nombre, València, 1800.

- J. Villanueva, Viage literario a las iglesias de España. La publica con algunas observa. ciones Joaquín Lorenzo Villanueva, Madrid, Imprenta Real, 1803.

${ }^{7}$ L. Nicolau d'OlWer, "La Cronica del Conqueridor i els seus problemes", Estudis Universitaris Catalans, XI, 1926, pp. 79m88.

${ }^{8}$ E. Martin-CHABot, "Pere Marsili et le Libre dels feyts del rey En Jacme lo Conqueridor", Bibliotheque de l'Ecole des Chartes, LXXII, 1911, pp. 92-99.

${ }^{9}$ Op, cit., 1996.

$10 \mathrm{~S}$. ASPERTI, "Indagini sull' Llibre dels feyts di Jaume I: Dall'originale all'archetipo", Rjb, 33, 1982, pp. 269-285.

11 Op. cit., p. 148, n. 5. 
Marsili y el Llibre dels fets tal como ha llegado a nosotros ${ }^{12}$, el punto de partida de nuestro trabajo es la consideración de la obra de Marsili como un caso de "recepción interlingüística"13 o, utilizando el término de G. Genette, un hipertexto del Llibre dels fets de Jaime $\mathrm{I}^{14}$.

\section{Planteamiento y Objetivos}

Como el propio Marsili explica en su prólogo y ha puesto de manifiesto el fino análisis que del mismo han hecho ya diversos estudiosos ${ }^{15}$, el objetivo del encargo de Jaime II de Aragón es dar difusión, en la todavía lengua internacional de cultura por excelencia, el latín, a la obra redactada en catalán que recoge las hazañas de su abuelo y que, hasta ese momento, había permanecido en los archivos de la casa real:

... racioni consonum in occulis illustrissimi domini lacobi, regis Aragonum, Valencie, Sardinie et Corsice, comitisque Barchinone, ac Sancte Romane Ecclesie uexillarii admirati et capitanei generalis, apparuit ut uictoriosissimi aui sui gesta pristinis temporibus, ueraci stilo sed uulgari collecta ac in archiuis domus regie ad perpetuam sue felicitatis memoriam reposita, reducerentur in medium, atque latino sermone diserta et per capitula iuxta conclusionum uarietatem distincta, unum historialem et cronicum redderent codicem, in quo tota dicta regis, aui sui, magnorum factorum texeretur series, et Deus in suo regali brachio ac gloriosissimo Principe a legentibus laudaretur. ${ }^{16}$

12 Nos hacemos eco aquí de las palabras de Soldevila (op. cit., p. 62) cuando afirma: "No em puc estar d'avançar, tanmateix, que no crec admissible la distinció: per a mi tot és la mateixa obra. [...] I, com en el cas de la Crònica del Ceremoniós, crec que, si realment és certa la teoria, les diferències entre el text premarsilià, diguem-ho així, de la crònica de Jaume I i el seu text postmarsilia, influit pel Marsili, degueren ésser molt poques, i en cap manera no justifiquen la distincio entre l'un i l'altre com dues obres diferents i amb designació o títol diferent." Una revisión reciente de ése y otros problemas de la obra puede encontrarse en el estudio introductorio de A. Ferrando y Vicent J. Escartí de su edición en catalán actual (Llibre dels fets. Estudi introductori i modernització lingüística per A. Ferrando i Vicent J. Escartí, València, Institució Alfons el Magnànim, 2008, pp. 23-32).

13 P. SAquero SuÁrez-SOMONTE, T. GonzÁlez Rol.Á, A. López Fonseca, La tradición clásica en España (siglos XIII-XV). Bases conceptuales y bibtiográficas, Madrid, 2002, p. 31.

14 Tal como lo define en Palimpsestos. La literatura en segundo grado, Madrid, Taurus, 1989 [1962] p. 17: "Llamo, por tanto, hipertexto a todo texto derivado de un texto anterior por transformación simple (diremos en adelante transformación sin más) o por transformación indirecta, diremos imitación."

15 Véase particularmente J. M. PujoL, "The Llibre del rei En Jaume: a Matter of Style", en A. DEYERMOND (ed.): Historical Literature in Medieval lberia, Londres, Department of Hispanic Studies/Queen Mary and Westfield College, 1996, pp. 35 65, concretamente en pp. 38 y ss.; R. VINAS, op. cit., pp. 210 y ss. o J. F. MESA, en su trabajo, aún en prensa, "Del Llibre dels fets a los Gesta lacobi de Fra Pere Marsili. Historiografía y propaganda", presentado en la Academia Valenciana de la Lengua en 2009.

16 La única edición del texto latino publicada hasta el momento es la de M. ${ }^{a} \mathrm{D}$. MarTínez SAN PEDro, op. cit., 1984, si bien aparecerá próximamente la que será la primera edición realmente "crítica" del texto, preparada por Dr. D. Antoni Biosca i Bas en el marco del Grupo de Investigación CODOLVA de la Universidad de Alicante. Por cortesía de su autor, para las citas utilizamos su texto -mucho más riguroso-, aunque aún no haya visto la luz. 
¿Cómo aborda el dominico esta tarea? Un simple cotejo revela que, en su objetivo de crear una obra historiográfica en latín a partir del relato autobiográfico de Jaime I, Marsili no siempre se ciñe escrupulosamente a su modelo y los estudiosos han señalado coincidencias y discrepancias entre ambas obras ${ }^{17}$ ) poniendo de relieve, particularmente, la tendencia de Marsili a amplificar el texto catalán introduciendo capítulos ausentes en el Llibre tales como, por ejemplo, la pormenorizada descripción de Mallorca (II, 1); el planctus por los Montcada (II, 22), el panegírico de Raimundo de Peñafort (IV, 47-49) o el excurso sobre los nombres de los vientos (II, 49) con el que trata de hacer gala de su erudición ${ }^{18}$.

Yendo un paso más allá de la constatación objetiva de las diferencias entre ambas obras, los críticos han hecho valoraciones muy diversas del trabajo de Marsili como traductor y, en ocasiones, claramente contrapuestas: con la consideración de Marsili como erudito prestigioso y capaz de un despliegue retórico ${ }^{19}$ contrastan juicios peyorativos como el de J. M. Pujol, que afirma que Marsili es "aware of his unpalatable latinity" 20 , habla del "seu llatí precari" 21 y califica su prosa como "rònega i utilitària"22, o el de Vinas, según el cual "il faut lire avec attention la Chronique de Marsili por «pêcher quelques perles", certes assez peu nombreuses"23.

Estos juicios de valor acerca de la prosa marsiliana, así como los que afirman lo contrario, a pesar de ser contradictorios entre sí, tienen en común el hecho de que están condicionados por la comparación con la crónica catalana, hasta el punto de que casi parecieran responder a una especie de proporcionalidad inversa en virtud de la cual el reconocimiento de los méritos literarios de una de las dos obras actuase en detrimento de los de la otra y, en nuestra opinión, esa vía de acercamiento al estudio de la versión latina no es adecuada.

El Llibre dels fets constituye, por sus muchas peculiaridades, un auténtico punto de inflexión en la tradición historiográfica catalana. Se trata de la crónica "del rey", en la que Jaime I se propone, como señala Pujol ${ }^{24}$, alecw cionar a sus sucesores, pero no a través de un tratado teórico, sino ofreciéndose a sí mismo como modelo. Por eso, en ella habla como protagonista;

17 Cf. Montoliu, 1952, op. cit.; Martinez SAN Pedro, op. cit., pp. 28-40; A. M. Badia I MarGARrT, "Punts de confluència entre la versió llatina i l versió catalana de la "Crònica" de Jaume I", Miscellània Joan Bastardas, vol. IV, Barcelona, PAM, 1990, pp. 15-24; J. M. Purol, op. cit., 1996, p. 41; R. VINAS, op. cit., 2004, p. 209; J. F. Mesa, que en su trabajo ofrece un cotejo comentado del libro IV de Marsili en relación con los capítulos correspondientes del Llibre dels fets y analiza la estructura del libro como conjunto.

18 Cf. Martin-Ciabor, op. cit., 1911, p. 98.

19 Por ejemplo, MarTin-Chabor, op. cit., 1911, pp. 95 y 98; Montoliv, op. cit., 1922, p. 178; Martinez SAN Pedro, op. cit., 1984, p. 44.

20 Op. cit., 1996, p. 41.

21 Op. cit., 2001, p. 158.

22 Op. cit., 2001, p. 159.

23 Op. cit., 2004 , p. 209.

24 Pujol, op. cit., 1996, p. 59: "Jaume I, wishing, like the other monarchs of his time, to indoctrinate his succesors in the royal function and the duties of kingship but not being prone to thinking in the terms suitable to written expression or at least not willing to inspire a theoretical tract, cast his advice in the form of a string of narrative autobiographical exempla." 
afirma que su intención es dejar constancia y memoria de sus propias hazañas, llevadas a cabo siempre bajo designio divino; se siente plenamente legitimado para narrar la historia de su reinado desde su propio punto de vista y se presenta como un elegido de la Providencia ${ }^{25}$.

Siendo esto así, el encargo de Jaime II de verter una obra tan personalísima al latín, a una lengua de cultura de carácter supranacional mucho más ajena al entorno personal del rey, es todo un reto para nuestro traductor ${ }^{26} \mathrm{y}$ su primera decisión (no sabríamos decir en qué medida elegida o impuesta) es aplicar como procedimiento de rescritura el cambio de narrador, de modo que el nós del texto catalán (a veces jo) pasa a ser en la versión latina rex o bien un pronombre singular de tercera persona. Como consecuencia de esta transposición, en la crónica de Marsili se diluye el punto de vista del rey y se nos desvanece esa impresión de viveza y frescura que percibimos en la crónica catalana, fuertemente marcada por la oralidad, como bien ha estudiado Pujol ${ }^{27}$. Veamos un ejemplo ilustrativo de este hecho, que va a condicionar en gran medida la presentación de los acontecimientos que hace Marsili:

§ 69: E, per tal que sàpian aquels qui hoiran aquest libre que cara cosa fo d’armes ço que feyt fo en Maylorques, deïm-vos-ne adés .I. carrera: que nuyl hom de peu, mariner ni altre, no osà jaure ab nós en la ost per .III. setmanes, sinó tan solament nós e.ls cavalers e.ls escuders qui.ns servien..$^{28}$

II, 24: Si quis qui hunc librum legerit fuit in quacumque obsidione ciuitatis, recordetur que uiderit, et istum exercitum plenum diuina gratia indicabit. Et ut rem maiori zelo plenam, maiori admiratione dignam uideat, et deffectus alibi recognoscat: certum est, et ipse bone memorie rex referre pluries consueuit, quod nullus pedestris, marinarius siue alius de nocte non iacuit in castris per tres septimanas, nisi solum rex, milites et scutiferi seruientes.

Del pasaje destacamos las siguientes observaciones:

1) Mientras que el rey da por hecho en este pasaje que su crónica va a ser recitada en voz alta y, más que leída, "escuchada" (hablamos, pues, de

25 Según explica A. M. COMPAGNA ("Comunicazione e propaganda nella storiografia catalana del secolo XIII", en R. CASTANO, F. LATELLA, T. SORRENTI (eds.), Comunicazione e propaganda nei secoli XII e XIII. Atti de convegno internazionale (Messina, 24-26 maggio 2007), Roma, 2007, pp. 217-222, concretamente en pp. 219-220), la Crónica de Jaime I marca distancias respecto de las otras crónicas catalanas, en las que la propaganda política no es en modo alguno tan explicita, ni se inserta tampoco en un discurso religioso, sino que se apoya en buena medida en la "letterarizzazione" del texto. Vid. también al respecto S. M. Cingolan, Historiografia, propaganda $i$ comunicació al segle XIII: Bernat Descolt $i$ les dues redaccions de la seva Cronica Barcelona, Institut d'Estudis Catalans 2006 y La memòria dels reis. Les Quatre Grans Cròniques i la historiografia catalana des del segle X fins al XIV, Barcelona, 2007.

${ }^{26}$ En ese sentido, podemos estar de acuerdo con Vinas cuando habla (2004: p. 211) de "La mission impossible de Pere Marsili".

27 Op. cit., 1996. Véase también al respecto J. BRUGuera, "La llengua del Ltibre dels fets. Una sintaxi marcada per l'oralitat", en G. COLÓN DOMÈnECh, T. MARTinez Romero (eds), op. cit., 2008, pp. 365-392.

${ }^{28}$ Las citas del texto catalán están tomadas de la edición de Jorpr Bruguera, Jaume , Llibre dels fets del rei En Jaume, a cura de Jordi Bruguera, 2 vols., Barcelona, Fundació Jaume I /Editorial Barcino, 1991. 
oralidad no sólo en la composición, sino también en la recepción ${ }^{29}$, Marsili sabe que su versión latina será, en todo caso, leída, y ello le lleva a cambiar el verbo.

2) Jaime I, buen conocedor de los avatares y peligros de la conquista de una ciudad, se permite exaltar el buen hacer de su ejército en el asedio de Mallorca, tanto porque tiene criterio para juzgar, como porque es consciente de que ese éxito le pertenece. Marsili, por su parte, apela al juicio del lector que tenga experiencia militar, ya que será el que sabrá valorar debidamente los méritos del ejército de Jaime I.

3) El rey, protagonista de los hechos que relata, ofrece un dato anecdótico extraído, obviamente, de su propia experiencia. Marsili, por contra, ha de justificar que conoce la anécdota y que es cierta, afirmando que se la había oído muchas veces contar al rey.

Así pues, como este pequeño ejemplo demuestra, resulta inevitable que el cambio de primera a tercera persona haga perder a la versión de Marsili inmediatez y autenticidad en comparación con el Llibre dels fets. Sin embargo, eso no significa que la crónica latina no tenga también su valor literario y, si queremos analizarla desde ese punto de vista para poder emitir juicios al respecto, no podemos limitarnos a compararla con el texto catalán sin más. Hemos de aproximarnos a la prosa de nuestro traductor tomando como referencia las características y modelos estilísticos del propio latín medieval, ya que solo desde esa pespectiva podemos valorar el nivel de elaboración al que Marsili somete su prosa.

En efecto, para el dominico, como para cualquier erudito medieval ${ }^{30}$, los textos escolares, constituidos muchas veces por florilegios y antologías de autores clásicos, forman un acervo común presente en su memoria. Marsili participa de ese entorno en el que circulan los textos literarios entre eruditos alimentados de la misma cultura e impregnados de las mismas lecturas, y nuestro objetivo en este breve trabajo es ofrecer una pequeña muestra de sus conocimientos literarios y de sus cualidades como prosista.

Para ello, vamos a detenernos en dos pasajes del libro II pertenecientes a la narración de los preliminares de la conquista de Mallorca, que es uno de los acontecimientos más gloriosos del reinado de Jaime I en tanto que significó su consolidación como monarca y el punto de arranque de la política expansionista que caracterizó su reinado. Esta parte de la obra nos parece particularmente atractiva ya que, a la gozosa exaltación que el Llibre dels fets hace de esta empresa, concebida y alentada por la Providencia divina, se suma la deliciosa evocación que, por su parte y alejándose de su modelo, hace Marsili de las tierras baleáricas y, especialmente, mallorquinas.

${ }^{30}$ Cf. P. Bourgan, Le latin médiévale, Turnhout, Brepols, 2005, p. 127. 


\section{Chronica Gestorum Iacobi I, II, 1 y II, 3: dos momentos de la con- quista de Mallorca}

\subsection{El descubrimiento de una realidad antes ignorada (II, 1)}

En este pasaje se dibuja por primera vez en el horizonte del rey la idea de Mallorca ${ }^{31}$ : Jaime I marcha a Tarragona y allí acuden también gran parte de nobles catalanes, a pesar de no haber sido convocados a Cortes. Pere Martell los invita a una cena en la que surge el tema de Mallorca y es el propio Martell, experto navegante, quien les habla de aquellas islas.

$\$ 47, \mathrm{E}$, passat mig any, nós fom a Terragona. E volch nostre Seyor que, meyns
de cort, que nós no havíem manada, foren ab nós la major partida dels nobles
de Cathalunya, e per nom: [...]. E convidà En Pere Martel, cuitadà de Barça-
lona e que sabia molt de mar, a nos e a tots aquels nobles qui eren aquí ab
nós. E, sus quant nós haguem prop de menjat, levaren-se paraules entre ells.
E dixem quiyna terra era Mayollorques ni quant té son regne. E demanaren-ho
a.N Pere Martel, per ço con era era comit de galees. E En Pere Martel dix-los
que.ls en diria noves, per ço car ja hi ha estat una vegada o dues.

II, 1: Omnipotente Deo, cuius prouidentia in sui disposicione non fallitur, ordinante, fere omnes nobiles Catalonie uenerunt ad regem, qui post medium annum a preteritis et supra inmediate in fine primi libri positis, uenerat Tarraconam. Nec tamen erant curie neque urgens quodcumque uniuersale aut commune negocium, sed propria cuiusque negocia et regis uisitatio affectata. Et ut de eis quos Deus scienter, ipsis nescientibus, ad hunc tam excellentem tractatum uocauerat, aliquos nominemus: [...] Et Petrus Martelli, ciuis Tarraconensis, artis nautice peritus, ipsum regem et dictos nobiles et audaciter inuitauit et sufficientissime procurauit. Pluribus itaque sibi inuicem succedentibus, cibariis iam fine apropinquante, posuit spiritus inter eos ut de insulis Catalonie propinquis et tunc temporis ualde nociuis, quas antiqui Baleares uocauerant, mencio fieret, principaliter de illa aliarum uicinarum matre, Mayorica, que piratis suis emissis frequentius dapna ingentia ipsius terre firme Catalanorum locis maritimis inferebat. Interrogant conuiue liberalem hospitem, et querunt a marine artis familiari qualis sit illa Mayoriqua, quanti spacii insula, que habeat fortalicia, et cetera huiusmodi. Refertque ille partim uisa partimque audita. Auditur attentius, et uultus et corda audientium ad eius relacionem ueridicam suspenduntur.

Como la simple lectura contrastada desvela, Marsili aplica de forma sistemática el procedimiento de la amplificación y pone de este modo particular énfasis en ciertas ideas o elementos. Veamos cuáles:

31 Antes de este pasaje, el nombre de "Maylorques" sólo aparece una vez, en una anticipación del rey que, al enumerar a los nobles que le aconsejaron el matrimonio con Leonor, menciona a Guillem de Montcada y recuerda que murió en Mallorca: "Y En Guillem de Muntcada, qui morí a Maylorques" $(18,15)$. Marsili reproduce la misma anticipación, incluyendo también a Raimundo de Montcada: "consentientibus Guillelmo de Montecatano et Raimundo de Montecatano, qui decesserunt Mayoricis" (I, 12). No obstante, J. M. Pusol (en R. VINAs (ed.), El Llibre dels fets de Jaume el Conqueridor. Versió en català modern de Josep Maria PuJot, Mallorca, Editorial Moll, 2008, p. 61) llama la atención sobre "la presentació novellesca que no es correspon amb la realitat" que en el Llibre hace del hecho el rey ya que, según un documento firmado por él con fecha de 23 de octubre, la decisión estaba ya tomada y el banquete aquí descrito tuvo lugar después, en torno a noviembre. 
1) En primer lugar, la actuación de la Providencia, que propicia ese encuentro en Tarragona, aparentemente fortuito, ya que los nobles acuden de forma espontánea sin haber convocado Cortes el rey. En el Llibre se menciona una vez ("E volch nostre Seyor que..."), pero Marsili incide en ello dos veces: una, al principio, en una mención mucho más explícita que la del texto catalán ("Omnipotente Deo, cuius prouidentia in sui dispositione non fallitur, ordinante...") y otra, ausente en el Llibre, que introduce justo antes de dar los nombres de los nobles que acudieron, y con la que anticipa la importancia de la empresa que se va a gestar, creando ya expectación en torno a la misma: "Et ut de eis quos Deus scienter, ipsis nescientibus, ad hunc excellentem tractatum uocauerat, aliquos nominemus...".

2) Marsili señala explícitamente el interés de los nobles por encontrarse con el rey como la única razón, junto con sus asuntos privados, que los había llevado allí: "Nec tamen erant curie neque urgens quodcumque uniuersale aut commune negocium, sed propria cuiusque negocia et regis uisitatio affectata". Al decir que no había "quocumque uniuersale aut commune negocium" que motivara la reunión, Marsili nos deja ver que ese asunto de alcance general va a nacer precisamente en ese encuentro, y a continuación recoge nuevamente el concepto en el "hunc excellentem tractatum" de la frase siguiente.

3) Cuando por primera vez se mencionan las islas Baleares, Marsili introduce una pequeña digresión (mucho más breve que la que hará después) en la que explica por qué esas islas son importantes y estratégicas para Cataluña y por qué entre ellas destaca Mallorca, a la que presenta como "illa aliarum uicinarum matre". El propósito de esta nueva anticipación es intensificar, en una gradatio ascendente, la expectación y el interés que poco a poco se suscita en torno a aquellas tierras.

4) En la versión latina el papel de Pere Martell en el desarrollo del banquete presenta matices peculiares. En el Llibre son los propios comensales (bien el rey, bien los nobles) ${ }^{32}$ los que muestran interés por saber cosas de Mallorca; recurren a Pere Martell pensando que él, "per ço con era còmit de galees", podría satisfacer su curiosidad. Él les dice que les puede responder "per ço car ja havia estat una vegada o dues" y, a continuación, les da información sobre las islas. El planteamiento es, pues, lógico y directo: Martell conoce por su oficio aquellas tierras y por eso puede hablar de ellas.

En Marsili, en cambio, el personaje es revestido de una importancia especial y el conjunto de la escena también. En primer lugar, es el propio Martell el que dirige la conversación de los comensales hacia ese tema

32 Al igual que en el caso de la identificación de la procedencia de Pere Martell, también sobre este punto hay discrepancias entre los manuscritos del Llibre: en la edición de Bruguera se adopta la lectura "dixem quiyna", que atribuye al rey la primera iniciativa en relación a la empresa de Mallorca. Los restantes manuscritos, en cambio, presentan "dixeren quina", poniendo la pregunta en labios de los nobles. Como explica Montoliu (op. cit., 1952, p. 8), esta lectura, que es la que sigue también Marsili, es la que resulta coherente con el subsiguiente discurso de los nobles ante Jaime I y el posterior desarrollo de los acontecimientos. 
("posuit spiritus inter eos ut de insulis Cataloniae propinquis [...] mentio fieret") ${ }^{33}$ y se recrea más explícitamente el contexto celebrativo del banquete. Pere Martell, el liberalem hospitem al que interrogan los convivae, se convierte así en el objeto de la atención de todos y, más que anfitrión, parece invitado, como la ambigua semántica del término latino hospes permite entender. Se le preguntan muchas cosas: "qualis sit illa Mayorica, quanti spacii insula, que habeat fortalicia, et cetera huiusmodi" y Martell atiende el requeriw miento de los nobles, pero no en su condición de navegante informado por la razón lógica de haber estado allí sin más, sino más bien como relator acerca de tierras lejanas, de cosas que en parte conoce por su propia experiencia, y que en parte le han contado: "Refertque ille partim visa, partim audita". La expresión evoca de forma inmediata un tópico característico del género paradoxográfico ${ }^{34} \mathrm{y}$, al utilizarla, Marsili otorga a Mallorca el rango de tema "tradicional", en el sentido más literal del término: todos quieren saber de aquellas tierras y su relato cautiva a su auditorio y mantiene en suspenso la atención de los oyentes: "Auditur attentius, et uultus et corda audientium ad eius relationem ueridicam suspenduntur". Esta atmósfera especial que crea Marsili en el momento de, por así decir, hacer entrar en escena la noción de la existencia de estas tierras lejanas contribuye, por supuesto, al mismo objetivo ya señalado de acrecentar la solemnidad del momento y resaltar la envergadura de la empresa que Dios ha decidido poner en manos de Jaime I, el elegido para este grandioso proyecto. No obstante, más allá de eso, cuando Marsili nos detalla las preguntas que le hacen a Pere Martell, cuando menciona la atenta escucha, los rostros y corazones de sus oyentes pendientes de sus palabras, tiene en mente una escena de la Eneida virgiliana, con la que crea un juego intertextual lleno de significación. Eneas, tras un largo periplo después de marcharse de Troya, arriba a las costas de Tiro y, acogido hospitalariamente en el palacio de Dido, es obsequiado con un banquete, en el que

33 Hay que decir que la lectura no resulta del todo clara. "Spiritus" puede tomarse como nominativo o como acusativo pero, tanto en el primer caso (el sujeto de "posuit" es el Espíritu divino, que es la interpretación que asumen VINAS (op. cit., 2004, p. 213) y QUADRADO (J. M. Quadrado, Historia de la conquista de Mallorca: crónicas inéditas de Marsili y de Desclot en testo lemosin. Vertida la primera al castellano y adicionada con notas y documentos por José María Quadrado. Palma 1850. [Hemos utilizado la edición electrónica publicada en la Biblio. teca Joan Lluís Vives: http:/www.lluisvives.com/FichaObra.html?Ref=4782\&portal=1]), como en el segundo (el sujeto es Pere Martell), alguien ajeno al rey y a los nobles es quien propicia que la conversación se encamine por esos derroteros. MARTínez SAN PEDro (op. cit., 1984, p. 155) ofrece un "posuit spatius", que no se explica bien y es, además, gramaticalmente incorrecto.

${ }^{34}$ En la narración de paradoxa, mirabilia, hechos sorprendentes y admirables y, por ello mismo, a veces increíbles, se alude frecuentemente al motivo de haber visto con los propios ojos como argumento para persuadir al receptor de la veracidad de lo narrado (cf. G. ScHEPENS, K. DELCROIX, "Ancient Paradoxography: origin, evolution, production and reception. Part II. The Roman Period", en O. PECERE, A. Stramaglia (eds.), La letteratura di consumo nel mondo grecoulatino. Atti del Convegno Internazionale, Cassino, 14-17 settembre 1994, Casino, Università degli Studi di Cassino, 1996, pp. 410 460, concretamente en p. 419). Vid., por ejemplo, Aulo Gelio V, 14, 2.4, IX, 4, 13. El género, nacido en la literatura helenística y cultivado también en las diferentes etapas de la literatura latina, será un terreno abonado para el posterior desarrollo de la literatura de viajes. 
la reina muestra gran interés por los avatares de la guerra y por la terrible suerte del pueblo troyano (Aen. I, 749-752) ${ }^{35}$ :

Infelix Dido longumque bibebat amorem

multa super Priamo rogitans, super Hectore multa;

nunc quibus Aurorae uenisset filius armis,

nunc quales Diomedis equi, nunc quantus Achilles.

Después, aún no satisfechos su curiosidad e interés, le pide a Eneas que relate desde el principio sus infortunios y los de los suyos. Se crea entonces una gran expectación entre los asistentes, pendientes de las palabras del héroe troyano: "Conticuere omnes intentique ora tenebant" (II, 1). El paralelismo entre Eneas que refiere su historia a requerimiento de la reina fenicia y Pere Martell que habla de Mallorca a invitación de los comensales se hace evidente por los obvios ecos verbales y pone de manifiesto la intencionalidad literaria de Marsili. Frente a la espontaneidad y a la inmediatez del recuerdo vivo del rey, el redactor latino aporta un artificio literario, un juego alusivo que impregna la escena de una particular grandeza a través de la evocación de un personaje que, siendo mítico, es real sin embargo en el universo cultural de Marsili. A continuación, frente a la rapidísima y escueta descripción de las islas que en estilo indirecto ofrece el Llibre, Marsili introduce, en labios del propio Martell, una digresión mucho más larga y detallada sobre las islas y, especialmente, sobre Mallorca, a la que, en gradación ascendente, reserva para el final como culminación de su enumeración y como punto y aparte respecto a las demás. Particularmente destacable es el tono entusiasta y emotivo con el que, por boca del navegante, ofrece Marsili al lector sus propios recuerdos y sensaciones: la amenidad de sus parajes, el clima templado y hospitalario, la riqueza de cultivos y ganados, la seguridad de sus puertos -acogedor refugio tras peligrosas y agotadoras travesías-, la fertilidad de los mares...

Sin entrar en un análisis detallado, sí queremos destacar la originalidad de esta creación exclusivamente marsiliana en la que quiasmos, similicadencias, enumeraciones y superlativos encomiásticos se suceden en una alabanza que recuerda en cierto modo la feliz y armoniosa convivencia de hombre y naturaleza, tópica en las descripciones clásicas de la mítica Edad de $\mathrm{Oro}^{36}$, pero que, sobre todo, recuerda la exaltación que hace Virgilio de las tierras itálicas en Geórgicas, II, 136 y ss. A este pasaje alude inequívocamente Marsili cuando después, ya como narrador (II, 1), para calificar las palabras de Martell sobre Baleares utiliza el término "laudibus Majoricarum" en clara alusión al "laudibus Italiae" virgiliano (Georg. II, 138).

${ }^{35}$ Citamos según la edición $\mathrm{P}$. Vergili Maronis, Opera, recognovit brevique adnotatione critica instruxit R. A. B. Mynors. Oxford, Oxford University Press, 1980 [1969].

${ }^{36}$ Vid., por ejemplo, Tibulo, 1, 3; Virgilio, Buc. IV; Georg. II 473-536; Horacio, Ep. XVI; Ovidio, Met. I, 89m150. 


\subsection{Jaime I, elegido por Dios para la conquista de Mallorca (II, 3)}

Tan sólo la crónica latina hace referencia explícita al grado sumo en que el relato de Martell sobre las Baleares sedujo a los nobles y cautivó sus ánimos (II, 2: "Summe placuit nobilibus et atraxit quod de laudibus Mayoricarum Petrus Martell retulit") pero, en cualquier caso, el resultado es el mismo: Jaime I recibe la petición unánime de los nobles de que se lance a la conquista del reino sarraceno y de esa tierra lejana que Dios decidió poner en medio del mar ("terra e regne dins en la mar, on Deus lo volch formar"; "unum regnum [...] et insulam talem et tantam quam Deus in medio mari statuit"). El consenso entre ellos es tal que el nombre de quién pronuncia el discurso se hace irrelevante ( $\$ 47$ : "E, quan aguem menjat, vengren denant nós e dixeren"; II, 2: "Et proponens unus coram aliis dixit"). El rey acepta y convoca Cortes generales en Barcelona para exponer el proyecto.

El momento es solemne y Marsili, para conferir aún mayor gravedad a las palabras de Jaime $\mathrm{I}$ en las Cortes (no menos importantes, obviamente, que las de Pere Martell en el banquete), trata ahora también de crear una cierta atmósfera de expectación e introduce los detalles, ausentes en el Llibre, de que el rey convocó Cortes sin decir el motivo ("non tamen negocium intimauit") y de que los convocados esperaban ávidos su discurso cuando se sienta en el trono real y toma la palabra ("figuntur in regem uultus omnium. Auide sunt aures ut audiant qua de causa tam solemnis curia celebretur"). El discurso, ciertamente, tiene un alto significado político y el particular énfasis que pone Jaime I en la protección y ayuda que Dios le concede para abordar esa empresa responde en buena medida a la necesidad de legitimar su liderazgo sobre unos nobles de los que amargas experiencias le habían enseñado que debía desconfiar.

§ 48: Illumina cor meum, Domine, et verba mea de Spiritu Sancto: On, nós pregam a nostre Senyor Déus e a la Verge sancta Maria, mare sua, que nós puxam dir algunes paraules que sían a honor de nós e de vós, qui les escoltarets, e que sien a placer de Déu e de la sua mare, nostra Dona sancta Maria. Car nós volem parlar de bones obres, car les bones obres vénen d'ell e só; e aqueles paraules que nós vos direm seran-ho. E plàssia a él que nós les puxam adur a acabament. Certa cosa és quel nostre naximent se féu per vertut de Déu, car no.s volien be nostre pare ni nostra mare, e sí fo volentat de Déu que nasquem en aquest món. $\mathrm{E}$ quan nós vos dixéssem les condicions ni les maraveyles que foren al nostre naxement, grans serían, mas lexar-nos em, per ço car al començament del libre se demostre. Mas ben sabem per cert que vós sabets que nós som vostre seyor natural, e som sols meyns de frare e de sor, que nostre pare no ach en nostra mare, e vingem entre vós jove, de jovén de.VI. ayns e mig, e trobam Aragó e Cathaluyna torbats, que los uns volien no u volien los altres; e havíets mala fama per lo món per les coses que eren passades. Aquest mal nós no podem adobar sinó per dues maneres, ço és, per volentat de Déu que -ns endreç en nostres affers, e que començem tals coses, e a vós e a nós, que a él vinga de plaer, e que la cosa sia tan gran e tan bona, que la mala fama que és entre vós que.s tolga, car la claror de les bones obres desfà l'escuredat. On, nós vos pregam molt carament per.II. raons, la primera per Déu, la segona er naturalea que nós havem ab vós, que vós que.ns donets conseyl e ajuda en .II. coses: la primera, que nós puscam nostra terra metre en pau; la segona, que nós puscam servir a nostre Seyor en est viatge que volem fer sobre.1 regne de 
Maylorques e les altres iles que pertanyen a aquela; la terça, que hajam conseyl de haver, en manera que aquest feyt puscam complir a honor de Déu.

II, 3: Quia omnia bona a Domino Deo nostro sunt, et sine eo neque uerba utilitatem habent neque opera uirtutem, rogamus humiliter Deum et Dominum nostrum Thesum Christum et gloriosam Virginem, eius matrem, quatinus nos, sua sapiencia illuminati, sua uirtute illustrati, possimus uobis que cogitauimus proponere, et uobiscum uerba ad opera taliter ordinare, quod sint ad laudem, honorem et gloriam filii et matris et exaltationem nostri regii diadematis, et ad leticiam cordis uestri. Magna enim et noua sunt que mente gerimus et omnipotenti Deo facilia, nostre autem potestati difficilia. Quare principaliter Deum inuocamus adiutorem et promotorem precipuum, et uestram prudentiam et consilium inuitamus. Audite, igitur, omnes diligencius, ut possitis melius respondere. Certum est quod aduentus noster in hunc mundum et corporalis natiuitas a Dei dono specialissimo fuisse concessus dinoscitur, quia, rege, patre nostro, suam consortem, reginam, habente odio, per magnam humanam -sed a Deo inspiratam- astuciam fuissemus generatus. Signa etiam et presagia plurima quasi de celo nobis arridente natiuitatis nostre tempore euenerent, per que hoc quod diximus de dono Dei confirmari uidetur. Nos sumus dominus uester naturalis et solus inter uos sine fratre aut sorore legitimus remansimus. Et uenimus ad regnandum super uos puer sex annorum et dimidii. Et inuenimus Cataloniam et Aragoniam in dispositione pessima, et terras multa superseminatas discordia omni pace et unitate uacuas, et multe iniquitatis amicas. Inde erat quod ex hiis et antea preteritis contra uos per mundum fama pessima laborabat. Huius igitur fame lese difusio ad plenum curari non poterit, nisi opera grandia Deo et mundo grata agrediamini, et magna Dei clementia uobis ad perficiendum assistat. Et tunc clarificabitur fama uestra sicut aeris obscuritas cedit, et ipse illuminatur cum sol ascenderit super terram. Contendite, igitur, uiriliter agere. Satagite Dei et uestrum ampliare nomen. Deponite uestem abhominabilem antiquate infamie, et ad noua et miranda opera unanimi animo consurgatis. Viam ad bonum uobis ostendimus. Magne uirtutis exercicium ministramus. Ecce enim nos, Deo nobis inspirante, Maioricas ire proponimus, et totum illud regnum Christo adquirere, nostrum dilatare dominium, et uestrum per uniuersum mundum ampliare nomen, sed et regem Maioricarum, tam infidelem, perfidum et damnosum uicinum, in uirtute Altissimi superare. Rogamus uos, igitur, primo Dei intuitu, cuius negocium agitur, secundo, naturalis amoris motu quem ad nostram habetis personam, quod in tribus nobis uestro consilio et adiutorio succurratis: primo, petimus per uos iuuari, ut, omnibus quibuscumque et inter quoscumque sedatis seditionibus, terram nostram in tranquilla pace possimus dimittere, qui nitimur adquirere alienas; secundo, petimus per uestrum consilium et adiutorium in isto negocio dirigi et foueri; tercio, petimus necessarium subsidium ut, dante Deo, uobiscum pariter de barbaris illis optata uictoria gaudeamus. Et hec sunt propter que uobis comunicanda et a uobis petenda ad nostras curias uocati uenistis.

La primera reflexión que cabe hacer al hilo de la lectura contrastada de ambos textos es que este caso Marsili, consciente quizá del alcance estratégico de las palabras del rey en este momento, no se permite introducir digresiones de su cosecha, si bien reorganiza en varios sentidos la ordenación de las ideas y aplica sistemáticamente, como es habitual en él, el procedimiento de la amplificación. Hay, no obstante, un caso de supresión que nos parece destacable: se trata de la praeteritio con la que el rey, cuando afirma que su 
nacimiento se debió a la voluntad divina, rehúsa extenderse en las circunstancias prodigiosas en que éste tuvo lugar y remite a la parte de su propia crónica en la que ya las ha relatado ("E quan nós vos dixéssem les condicions ni les maraveyles que foren al nostre naxement, grans serían, mas lexar-nos em, per ço car al començament del libre se demostre"). La remisión a los primeros capítulos del Llibre es fruto del estilo espontáneo y directo que lo caracteriza: Jaime I no pierde de vista en ningún momento que está contando sus memorias y, sabedor de que ya ha hablado de ese asunto en su crónica, evita repetirse a sí mismo y alargarse demasiado sobre un tema que ya ha sido narrado. Aquí no se está dirigiendo a su auditorio en las Cortes, sino al oyente o lector de su crónica, y es que, para él, los límites entre las palabras que pronunció en la sesión de Cortes y las que quiere reproducir en el Llibre son difusos, ya que en todo momento es él quien habla de sí mismo. Marsili, en cambio, trata de evitar esta interferencia en el discurso porque él pretende elaborar una pieza oratoria que, siguiendo la tradición de los discursos de historiógrafos clásicos como Salustio, Tito Livio o Tácito, sea, por un lado, artísticamente elaborada y, por el otro, verosímil, en tanto que reproduzca las palabras que hubiera podido pronunciar el personaje en cuestión en ese momento histórico. Por ello sustituye la frase por una redundante referencia a una expresión utilizada por él mismo unas líneas más arriba:

Certum est quod aduentus noster in hunc mundum et corporalis natiuitas a Dei dono specialissimo fuisse concessus dinoscitur [...]. Signa etiam et presagia plurima quasi de celo nobis arridente natiuitatis nostre tempore euenerent, per que hoc quod diximus de dono Dei confirmari uidetur.

Por otro lado, considera insuficiente la escueta mención de la expedición militar a las Baleares que, siendo supuestamente el tema principal, no es mencionada, sin embargo, hasta casi el final del discurso catalán, sin preámbulo alguno, como segundo de los tres puntos en los que Jaime I pide consejo y ayuda ("...la segona, que nós puscam servir a nostre Seyor en est viatge que volem fer sobre.l regne de Maylorques e les altres iles que pertanyen a aquela"). Para darle mayor relieve a la idea, Marsili la anticipa con varias alusiones más o menos veladas que no están en su modelo ("uobis que cogitauimus proponere"; "Magna enim et noua sunt que mente gerimus"; "ad nou et miranda opera unanimi animo consurgatis") y, después, la menciona explícitamente, pero no como concepto subordinado a la especificación de los puntos en los que el rey solicita ayuda, sino como una frase principal en la que pone el acento, por medio de una notable amplificatio, en el carácter de cruzada que tiene la empresa:

Ecce enim nos, Deo nobis inspirante, Maioricas ire proponimus, et totum illud regnum Christo adquirere, nostrum dilatare dominium, et uestrum per uniuersum mundum ampliare nomen, sed et regem Maioricarum, tan infidelem, perfidum et damnosum uicinum, in uirtute Altissimi superare.

Nuestro traductor intenta, pues, darle fuerza persuasiva al discurso y crear una pieza oratoria convincente y "viva" que responda al objetivo, no tanto de instruir (docere/probare) o agradar (delectare), cuanto al de conmo- 
ver (mouere/flectere) que, dentro de la teoría retórica, es el más ambicioso de los officia oratoris y el más adecuado a un tema solemne y a un estilo elevado ${ }^{37}$. Por ello, introduce varios imperativos en diferentes momentos del discurso con los que pretende renovar la atención de su auditorio y sacudir de alguna manera sus conciencias a favor de la empresa que propone:

Audite, igitur, omnes diligencius, ut possitis melius respondere.

Contendite, igitur, uiriliter agere; satagite Dei et uestrum ampliare nomen.

Deponite uestem abominabilem antiquate infamie et ad noua et miranda opera unanimi animo consurgatis.

Se trata, obviamente, de un recurso artificioso que difícilmente puede sustituir la fuerza y la autoridad que emanan de la figura del rey cuando en el discurso catalán hace ver a su auditorio que él es el elegido de Dios para una empresa que se debe más a la voluntad divina que a la suya propia y que es ayudándole a él como podrán recuperar el favor divino y limpiar la ignominia de sus anteriores acciones. Pero, así como a Jaime I le preocupa sobre todo insistir en su peculiar vínculo con Dios, como sublime legitimación de su autoridad ("E plàssia a él que nós les puxam adur a acabament"), Marsili, interesado como hemos dicho en la verosimilitud del discurso como tal, intenta además reforzar el vínculo entre orador y público y añade pequeñas referencias a lo largo de toda la alocución (la primera de ellas ya en el mismo exordio y la última a modo de peroratio) que establecen una relación más directa y una complicidad más explícita con el auditorio:

- Rogamus humiliter Deum [...] quatinus nos [...] possimus uobis que cogitauimus proponere et uobiscum uerba ad opera taliter ordinare quod...

- Quare principaliter Deum inuocamus adiutorem et promotorem precipuum et uestram prudentiam et consilium inuitamus.

- Viam ad bonum uobis ostendimus

- Ut, dante Deo, uobiscum pariter de barbaris illis optata uictoria gaudeamus

- Et hec sunt propter que uobis comunicanda et a uobis petenda ad nostras curias uocati uenistis.

Así pues, Marsili pone una particular atención, tanto en la recreación de la actio del discurso, con las frases preliminares que, según dijimos, tratan de crear una cierta expectación, como en la eficacia persuasiva del mismo, demarcando claramente sus límites dentro del relato y tratando de sumar, en lo que respecta a la figura de Jaime I, la autoridad de orador a la autoridad de rey.

37 Vid. Cic., Or, XXI, 69-70: "Erit igitur eloquens - hunc enim auctore Antonio quaerimus - is qui in foro causisque civilibus ita dicet, ut probet, ut delectet, ut flectat. Probare necessitatis est, delectare suavitatis, flectere victoriae: nam id unum ex omnibus ad obtinendas causas potest plurimum. Sed quot officia oratoris, tot sunt genera dicendi: subtile in probando, modicum in delectando, vehemens in flectendo; in quo uno vis omnis oratoris est. Magni igitur iudici, summae etiam facultatis esse debebit moderator ille et quasi temperator huius tripertitae varietatis; nam et iudicabit quid cuique opus sit et poterit quocumque modo postulabit causa dicere. Sed est eloquentiae sicut reliquarum rerum fundamentum sapientia. Vt enim in vita sic in oratione nihil est difficilius quam quid deceat videre. Prepon appellant hoc Graeci, nos dicamus sane decorum; de quo praeclare et multa praecipiuntur et res est cognitione dignissima". 
Por otro lado, el traductor latino hace también un notable esfuerzo por enriquecer el discurso con un despliegue de recursos formales que contrastan claramente con la prosa mucho más sencilla y plana de que hace gala en otros pasajes. En un estilo hipotáctico con gran abundancia de subordinación, Marsili trata de alejarse del orden de palabras natural de las lenguas romances y se esfuerza en seguir el orden SOV propio del latín clásico estableciendo marcadas correspondencias entre los diversos sintagmas a través de paralelismos, antítesis y quiasmos: "Sine eo neque uerba utilitatem habent neque opera uirtutem"; "sua sapiencia illuminati, sua uirtute illustrati"; "omnipotenti Deo facilia, nostre autem potestati difficilia"; "terras multa superseminatas discordia, omni pace et unitate uacuas et multe iniquitatis amicas"; "opera grandia Deo et mundo grata".

Junto a figuras propias del ornatus difficilis ${ }^{38}$, como la metáfora del ropaje de la infamia ("uestem abominabilem antiquate infamie") o la del favor divino ante el nacimiento del rey ("de celo nobis arridente"), Marsili utiliza, además, el recurso estilístico del cursus rhythmicus ${ }^{39}$, la característica más destacada por la que se define el stylus gregorianus ${ }^{40}$, y emplea secuencias rítmicas, tanto a final de periodo, como en interior de frase, con una marcada preferencia por el cursus uelox. Así, por ejemplo: Táliter ordináre (C.V.); Déo facília (C.T.); promotórem precípuum (C.T.); consílium inuitámus (C.V.); mélius respondére (C.V.); concéssus dinóscitur (C.T.); fuissémus generátus (C.Disp.); confirmári uidétur (C.P.); iniquitátis amícas (C.P.); péssima laborábat (C.V.); perficiéndum assístat (C.P.); antiquáte infámie (C.T.); ánimo consurgátis (C.V.); exercícium ministrámus (C.V.); íre propónimus (C.T.); damnósum uicínum (C.P.); Altíssimi superáre (C.V.); ad nostram habétis persónam (C.P.); adiutório succurrátis (C.V.); uictória gaudeámus (C.V.); uocáti uenístis (C.P.).

38 Cf. Bourgaln, op. cit., 2005, pp. 394-5.

39 Sobre los diferentes tipos de cursus así como sobre su génesis y uso en diversos ámbitos y autores, pueden consultarse, entre otros, M.G. Nrcolau, Lorigine du cursus rythmique et les débuts de l'accent d'intensité en latin, Paris, 1930; H. HAGENDAKt, La prose métrique d'Arnobe. Contributions à la connaissance de la prose littéraire de l'Empire, Göteborg, 1937; G. LiNDHoLM, Studien zum Mittelateinischen Prosarhythmus, Stockholm, 1963; M. PLEZIA, "Lorigine de la theorie du cursus rythmique au XII siècle", ALMA, 39, 1974, pp. 5-22; T. JANSON, Prose rhythm in Medieval Latin from the $9^{\text {th }}$ to the $13^{\text {th }}$ century, Stockholm, 1975; S. M. OBERHELman, R. G. Hal., "A New Statistical Analysis of Accentual Prose Rhythms in Imperial Latin Authors", $C P, 79$, 1984, pp. 114 130; "The cursus in Late Imperial Latin Prose: a Reconsideration of Methodology", $C P, 83,1988$, pp. 136 149; "The History and Development of the cursus mixtus in latin Literature", CQ, 38, 1988, pp. 228 242; A. F. MEMOLI, Studi sulla prosa d'arte negli scrittori cristiani, Napoli, 1979; G. LORETEGU, "El uso del cursus en algunos privilegios de la cancillería de Sancho el Sabio", Principe de Viana n.. 205, 1995, pp. 509-526.

$40 \mathrm{El}$ stylus gregorianus, que debe su nombre a uno de los más importantes teóricos del cursus, Alberto di Morra, futuro Papa Gregorio VIII, es uno de los cuatro estilos básicos de la prosa que, a principios del siglo XIII, diferencia Juan de Garlande en su Parisiana poetria en función de diversos elementos formales que predominan en cada uno de ellos. Junto al gregorianus, marcado por el uso del cursus, señala el stylus tullianus, caracterizado por el uso abundante de figuras retóricas, el stylus hilarianus, que imita la estructura rítmica del himno, y el stylus isidorianus, caracterizado por la rima (cf. F. A. C. MANTELlo, A. G. RIGG, Medieval Latin. An Introduction and bibliographical Guide, Washington, D.C., The Catholic University of America Press, 1996, p. 114; Bourgain, op. cit., 2005, p. 397). 
A la luz de todos estos datos podemos decir que Marsili, al elaborar la versión latina del discurso del rey ante las Cortes, la somete a un mayor grado de elaboración formal y, actuando más como orator que como simple interpres $^{41}$, se consagra no tanto a la inuentio, cuanto a la dispositio, modificando en varios sentidos la ordenación de las ideas, y a la elocutio, tratando de elevar el estilo de su prosa y adecuarlo a la solemnidad del tema, tal como aconseja el precepto del decorum ${ }^{42}$.

\section{Recapitulación}

Nos proponíamos al principio de este trabajo aproximarnos a la prosa de Marsili cambiando la perspectiva desde la que se ha juzgado tradicionalmente su prosa, de modo que el punto de referencia para valorarla no sea tanto el Llibre dels fets, cuya originalidad, viveza y frescura no tienen parangón, cuanto las convenciones estéticas propias de la prosa latina medieval y los modelos clásicos que forman parte inalienable de un acervo cultural del que Marsili, como erudito medieval, participa.

El análisis que hemos realizado de dos pasajes correspondientes a la narración de los preliminares de la conquista de Mallorca pone de manifiesto que nuestro traductor, sensible a la importancia que ya la crónica catalana concede al episodio, no se limita a una traducción literal, sino que introduce digresiones completamente originales, refuerza la visión providencialista de su modelo e introduce elementos estilísticos y literarios nuevos, ajenos al mundo recreado en el Llibre dels fets, con los que trata, por un lado, de conferir majestad a los acontecimientos que relata y, por el otro, de elevar el estilo para adecuarlo a la grandiosidad de unos proyectos que, en palabras del arzobispo de Tarragona cuando responde al discurso del rey, son "laude digna diuina pariter et humana"43.

En estos pasajes, al igual que en otros muchos a lo largo de la crónica, Marsili busca embellecer y conferir elegancia a su prosa y para ello utiliza juegos intertextuales, figuras retóricas o secuencias rítmicas del cursus, todos ellos recursos de ornatus que quedan al descubierto solamente cuando se analiza su crónica en relación con la amplia tradición literaria latina en la que ésta se inserta.

Quizá, el efecto resultante de este trabajo de elaboración formal es el de una prosa artificiosa que contrasta fuertemente con la frescura de la crónica real y, ciertamente, en el nuevo camino literario que toma la historiografía

41 Retomando la ya conocida diferencia que establece Cicerón entre ambos conceptos en De opt. gen. or, V 14: "Converti enim ex Atticis duorum eloquentissimorum nobilissimas orationes inter seque contrarias, Aeschinis et Demosthenis; nec converti ut interpres, sed ut orator, sententiis isdem et earum formis tamquam figuris, verbis ad nostram consuetudinem aptis. In quibus non verbum pro verbo necesse habui reddere, sed genus omne verborum vimque servavi, Non enim ea me adnumerare lectori putavi oportere, sed tamquam appendere".

${ }^{42}$ Vid. Cicerón, Or. XIX 100: «is est enim eloquens qui et humilia subtiliter et magna grauiter et mediocria temperate potest dicere» y también supra, n. 37 .

43 II, 9: "Viso enim quid intenditis, quid petitis, dicimus quod omnia sunt laude digna diuina pariter et humana". 
catalana ${ }^{44}$, una versión latina del Llibre dels fets parece encontrar difícil acomodo. Sin embargo, este hecho no debe ser obstáculo para reconocerle a Marsili unos conocimientos literarios y unas cualidades de escritor que ya esta breve aproximación pone de manifiesto y que un estudio estilístico más detallado y completo del conjunto de su obra nos permitirá ponderar adecuadamente.

ABstract: In this article we have analyzed two fragments relating to the conquest of Mallorca found in Pere Marsili's Latin translation, ordered by Jaume II, of the Llibre dels Fets by Jaume I: the banquet in Tarragona and the King's speech in the Parliament of Barcelona. The comparative analysis shows that Marsili did not limit himself to making a literal translation, but included original digressions, reinforced the providentialist outlook of his model and made use of a high level of literary elaboration in his prose availing himself of intertextual references, numerous rhetoric figures or rhythmic sequences of the "cursus" so as to adapt his style to the solemnity and magnificence of the acts he was relating.

KEY woRDS: medieval Latin; Jaume I; Marsili.

44 Ese punto de inflexión que representan las crónicas catalanas lo expresa muy bien AURELl (op. cit., 2005, p. 263): "The reign of Jaume I el Conqueridor marked a real turning point in medieval Catalan history and historiography. The earlier political fragmentation had pervented the consolidation of historiographic culture. In the thirteenth century, Catalan historiography developed from being a complied history to a composed history, from an officious history to an official history, from a monastic history to a courtly history, from an anonymous history to an author's history, from a schematic history to a narrative history, from a poetic history to a narrative history, from a Latin history to a vernacular history, from a genealogical history to a chronicle history." 